OPEN ACCESS

Edited by:

Shreerang Bhide,

Institute of Cancer Research (ICR),

United Kingdom

Reviewed by:

Derfel Ap Dafydd,

Royal Marsden Hospital,

United Kingdom

Avraham Eisbruch,

University of Michigan, United States

*Correspondence:

Steve Connor

steve.connor@kcl.ac.uk

Specialty section:

This article was submitted to

Head and Neck Cancer.

a section of the journal

Frontiers in Oncology

Received: 28 September 2021 Accepted: 13 January 2022

Published: 15 February 2022

Citation:

Guha A, Anjari M, Cook G, Goh V and Connor S (2022) Radiomic Analysis of Tumour Heterogeneity Using MRI in

Head and Neck Cancer

Following Chemoradiotherapy:

A Feasibility Study.

Front. Oncol. 12:784693.

doi: 10.3389/fonc. 2022.784693

\section{Radiomic Analysis of Tumour Heterogeneity Using MRI in Head and Neck Cancer Following Chemoradiotherapy: A Feasibility Study}

\author{
Amrita Guha ${ }^{1,2,3}$, Mustafa Anjari ${ }^{4}$, Gary Cook $^{3,5}$, Vicky Goh ${ }^{3,4}$ and Steve Connor ${ }^{3,4,6 *}$ \\ 1 Department of Radio-Diagnosis, Tata Memorial Hospital, Mumbai, India, 2 Training School Complex, Homi Bhabha National \\ Institute, Mumbai, India, ${ }^{3}$ School of Biomedical Engineering \& Imaging Sciences, Faculty of Life Sciences \& Medicine, \\ King's College London, London, United Kingdom, ${ }^{4}$ Department of Radiology, Guy's and St Thomas' Hospital, London, \\ United Kingdom, 5 King's College London \& Guy's and St Thomas' Positron Emission Tomography (PET) Centre, London, \\ United Kingdom, ${ }^{6}$ Department of Neuroradiology, King's College Hospital, London, United Kingdom
}

Objectives: To evaluate interval changes in heterogeneity on diffusion-weighted apparent diffusion coefficient (ADC) maps and T1-weighted post-gadolinium (T1w post gad) MRI in head and neck carcinoma (HNSCC), with and without chemo-radiotherapy (CRT) response.

Methods: This prospective observational cohort study included 24 participants (20 men, age $62.9 \pm 8.8$ years) with stage III and IV HNSCC. The primary tumour $(n=23)$ and largest lymph node $(n=22)$ dimensions, histogram parameters and grey-level co-occurrence matrix (GLCM) parameters were measured on ADC maps and T1w post gad sequences, performed pretreatment and 6 and 12 weeks post CRT. The 2-year treatment response at primary and nodal sites was recorded. The Wilcoxon signed-rank test was used to compare interval changes in parameters after stratifying for treatment response and failure $(p<0.001$ statistical significance).

Results: 23/23 primary tumours and 18/22 nodes responded to CRT at 2 years. Responding HNSCC demonstrated a significant interval change in ADC histogram parameters (kurtosis, coefficient of variation, entropy, energy for primary tumour; kurtosis for nodes) and T1w post gad GLCM (entropy and contrast in the primary tumour and nodes) by 6 weeks post CRT ( $p<0.001)$. Lymph nodes with treatment failure did not demonstrate an interval alteration in heterogeneity parameters.

Conclusions: ADC maps and T1w post gad MRI demonstrate the evolution of heterogeneity parameters in successfully treated HNSCC by 6 weeks post CRT; however, this is not observed in lymph nodes failing treatment.

Advances in Knowledge: Early reduction in heterogeneity is demonstrated on MRI when HNSCC responds to CRT.

Keywords: radiomics, heterogeneity, head and neck cancer, chemoradiotherapy, texture analysis, magnetic resonance imaging 


\section{INTRODUCTION}

Head and neck squamous cell carcinoma (HNSCC) is the seventh most common cancer worldwide (1). Chemoradiotherapy (CRT) provides the best opportunity for cure in advanced-stage HNSCC (2); however, tumour resistance or insufficient therapy may result in treatment failure in more than $30 \%$ of patients (3). Whilst earlier detection of residual viable tumour allows for salvage surgery and improved survival (4), it is currently challenging to evaluate this with clinical examination and cross-sectional imaging, due to the presence of posttreatment tissue distortion (5). Metabolic imaging with 18F-fluorodeoxyglucose positron emission tomography/ computed tomography $\left({ }^{18} \mathrm{~F}-\mathrm{FDG}\right.$ PET/CT) (6) may overcome some of the difficulties in interpretation with conventional CT and MRI in this clinical context, but it is generally delayed for at least 12 weeks, due to earlier false positives from post-CRT inflammation (7).

Assessing changes in MRI signal heterogeneity within tumours $(8,9)$ may better reflect residual disease. There are limited studies addressing the application of such analysis of imaging features in the prediction of treatment response $(10,11)$, and there are no data on their early posttreatment evolution in HNSCC. It is well accepted that HNSCCs display a marked "heterogeneity" on histology with variation in proliferation and cellular differentiation within different regions of the tumours $(12,13)$. We hypothesised that alterations in tumour heterogeneity following CRT would be reflected by diffusionweighted and post-gadolinium T1w MRI and that measurements of signal heterogeneity may augment standard response assessment based on size criteria alone.

Therefore, our primary objective was to evaluate the interval changes in signal heterogeneity on diffusion-weighted (DWI) apparent diffusion coefficient (ADC) maps and T1-weighted post-gadolinium (T1w post gad) MRI within the primary tumour and largest metastatic lymph node at 6 and 12 weeks following CRT. Our secondary objectives were to evaluate how interval changes vary according to treatment response and to compare the interval changes in parameters to those of conventional size criteria.

\section{MATERIALS AND METHODS}

\section{Study Design}

Participants were recruited for a prospective single-centre cohort observational study (ISRCTN58327080; Research Ethics Committee approval 13/LO/1876) and provided informed consent for participation.

\section{Participants}

Participants were eligible if there was histologically confirmed stage III or IV primary squamous cell carcinoma of the head and neck (HNSCC) without distant metastatic disease, a $1-\mathrm{cm}^{2}$ area of measurable primary tumour and/or metastatic locoregional node on the basis of standard clinico-radiological staging, and where curative CRT was planned. Exclusion criteria were prior chemo- or radiotherapy; ECOG performance status $>2$; lack of capacity to provide informed consent; and known contrast agent allergy or renal impairment.

\section{Treatment}

Intensity-modulated radiotherapy consisting of $70 \mathrm{~Gy}$ in 35 fractions was delivered, 2 Gy per fraction once daily, 5 days a week. Concomitant intravenous cisplatin at a dose of $35 \mathrm{mg} / \mathrm{m}^{2}$ every 7 days, starting on day 1 of radiotherapy, was administered to all participants.

\section{MRI Imaging}

Participants underwent MRI before treatment and at 6 and 12 weeks after completion of chemoradiotherapy. Imaging was performed on a 1.5-Tesla MRI system (MAGNETOM Aera, Siemens Healthcare, Erlangen, Germany) using a 20-channel phased-array surface neck coil and included T2-weighted, diffusion-weighted imaging (DWI) and post gad T1w sequences. The MRI acquisition protocol is summarised in Supplemental Table 1.

\section{MRI Processing and Analysis}

Two sets of freehand regions of interest (ROI) were delineated by a radiologist (AG, 7 years of experience) on both DWI $(b=800)$ and post gad T1w images with reference to the other sequences. ROIs were placed at the site of the measurable primary tumour and/or largest pathological lymph node on the pretreatment, 6-week and 12-week post-treatment MRIs. ROIs were placed on multiple sections to encompass the whole lesion depicted as high signal on the DWI b-800 or high signal on post gad T1-w sequences (Figure 1). Internal areas of necrosis (non-enhancement and high diffusion signal, respectively) were included, but areas of peritumoural inflammation (adjacent avid gadolinium enhancement and high signal on $\mathrm{T} 2 \mathrm{w}$ images) were excluded. When a focus of increased signal relative to background was not evident on the postCRT imaging, this was defined as non-measurable, and a representative ROI $\left(6-\mathrm{mm}\right.$ diameter $\left./ 28 \mathrm{~mm}^{2}\right)$ was placed at the site of the pretreatment ROI. For DWI, the ROI was transferred onto the ADC maps calculated from the $b=100$ and $b=800$ values to derive ADC parameters. In patients where it was challenging to draw ROIs on the posttreatment scans, the $\mathrm{T} 2 \mathrm{~W}$ and $\mathrm{T} 1$ postgadolinium images were also correlated with ensuring the accuracy of delineation.

Primary tumour and nodal histogram and grey-level cooccurrence matrix (GLCM) parameters were extracted from the ROIs using an in-house radiomics software platform, validated through the International Biomarker Standardization Initiative (14). Analysis was restricted to these parameters to reflect the limitations of the $2 \mathrm{D}$ acquisition and in-plane voxel resolution. For example, GLCM analysis was only applied to post gad T1w images since the larger voxel size $(0.9 \times 0.9 \times 5 \mathrm{~mm})$ of the diffusion acquisition resulted in insufficient voxels for analysis.

The mean and median gadolinium-enhanced T1w signal and $\mathrm{ADC}$ value were also recorded as well as the lesion long axis length and volume on the post gad T1w images. 


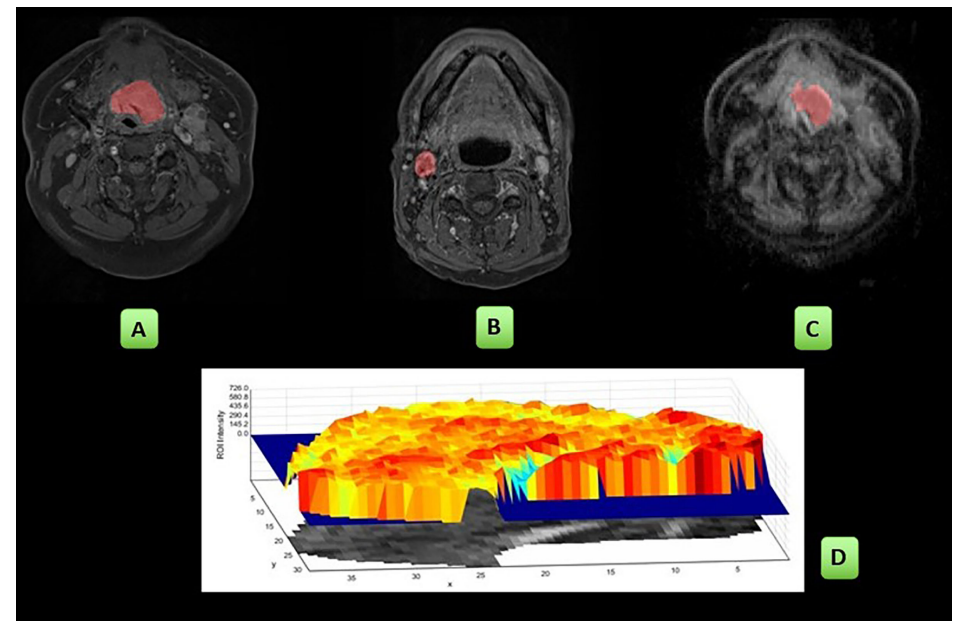

FIGURE 1 | Workflow of the study: ROls were placed on the post gad T1w sequences: primary tumour (A), single largest pathological lymph node (B) and on the ADC map: primary tumour (C). The radiomics first- and second-order parameters were derived and are pictorially depicted here by a sample of the shaded surface intensity histogram (D). These analyses were then repeated at the 6- and 12-week MRI studies.

\section{Clinical Data and Treatment Outcome}

Patient demographics, TN status, human papillomavirus (HPV) status, tumour site and subsite tumour stage were recorded. Followup included a standard-of-care 12-week 18F-FDG PET/CT study which was used to guide management and clinical assessment at 1 year and 2 years following completion of chemo-radiotherapy. The response of primary tumour and largest lymph node at 2 years was determined by cytological or histological confirmation (biopsy or resection) or by serial progression on imaging follow-up.

\section{Statistical Analyses}

SPSS statistical software Version 21.0 was used for analysis (IBM SPSS Statistics for Windows, Version 21.0. Armonk, NY: IBM Corp. Released 2012).

Pearson's correlation was performed as a method of parameter reduction amongst the initial 21 GLCM parameters, excluding those with a high correlation coefficient $(>0.75)$. This left 4 GLCM parameters for further analysis (Supplemental Table 2).

Descriptive data were collated for pretreatment, 6-week and 12-week post-CRT histogram and the selected GLCM parameters. This was performed separately for primary tumour and lymph node values and according to whether there was treatment response at 2 years.

As variables were not normally distributed, the Wilcoxon signed-rank test was performed to assess the interval changes in heterogeneity parameters. A p-value of $\mathrm{p}<0.001$ was considered statistically significant to account for multiple testing.

\section{RESULTS}

\section{Participants}

The participant consort flow diagram is demonstrated in Figure 2. Of the 24 participants ( 20 men, 4 women, mean age
$62.9+8.79$ years), there were $5(20 \%)$ patients with stage III disease and 19 (79\%) patients with stage IV disease. Participant characteristics including primary site, HPV status and TN staging are summarised in Table 1. There were 23 primary tumours and 22 lymph nodes analysed.

At 6 weeks, there were 19/23 non-measurable primary tumours and 10/22 non-measurable nodes whilst at 12 weeks there were $23 / 23$ non-measurable primary tumours and $18 / 22$ non-measurable nodes. All primary tumours and 18/22 lymph nodes responded to CRT at 2 years. The 4 cases of lymph node recurrence corresponded to the same largest lymph node site as that undergoing analysis. Patients with non-responding lymph nodes underwent salvage neck dissection following initial cytological assessment.

\section{Interval Changes Following CRT in the Primary Tumour}

All primary tumours responded to CRT at 2 years following treatment.

The primary tumour showed a significant mean decrease of $71 \%\left(6.3+\_0.8 \mathrm{~cm}\right)$ in diameter and $98 \%\left(1.45+\_0.5 \mathrm{~cm}^{3}\right)$ in volume at 6 weeks and a further reduction between 6 and 12 weeks $(\mathrm{p}<.0001)$

There was a significant increase in mean values of ADCmean and ADCmedian (729 to $1,019 \times 10^{-6} \mathrm{~mm}^{2} / \mathrm{s} ; 40 \%$ and 692 to $1,062 \times 10^{-6}$ $\mathrm{mm}^{2} / \mathrm{s} ; 53 \%$ ) by 6 weeks post CRT, but there was no further significant increase between 6 and 12 weeks. Kurtosis (-42\%), coefficient of variation (CoV) (-46\%) and entropy (-44\%) significantly decreased, and energy significantly $(+238 \%)$ increased by 6 weeks of treatment $(\mathrm{p}<.0001)$, again without further significant alterations between 6 and 12 weeks post CRT (Table 2).

None of the histogram parameters showed any significant changes on post gad T1w images at 6 or 12 weeks. GLCM contrast significantly increased $(+106 \%)$ and GLCM entropy 


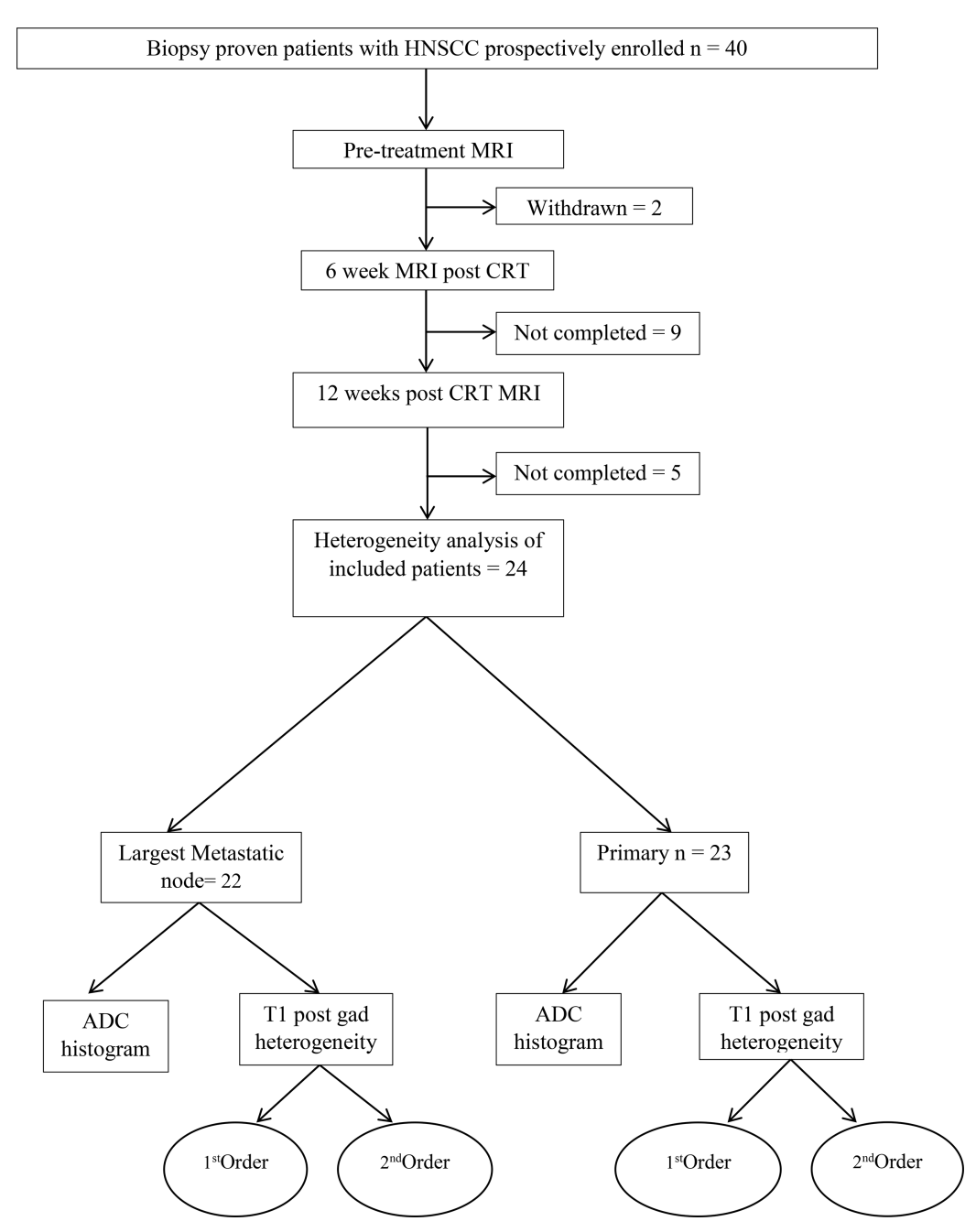

FIGURE 2 | Patient flowchart.

TABLE 1 | Participant characteristics of the HNSCC cohort.

\begin{tabular}{llc}
\hline Gender & Male & 20 \\
& Female & 4 \\
Age (years) & Mean & \\
& Median & 62.95 \\
& Range & 65 \\
& & $47-77$ \\
Tumour location & Oropharynx (all HPV positive) & \\
& Hypopharynx & 17 \\
T stage & Laryngopharynx & 3 \\
& T1 & 4 \\
& T2 & 5 \\
N stage & T3 & 7 \\
& T4 & 6 \\
& No & 6 \\
& N1 & 2 \\
& N2a & 5 \\
Human papillomavirus status & N2b & 1 \\
& N2c & 12 \\
& Positive & 4 \\
& Negative & 21 \\
& &
\end{tabular}

(-36\%) significantly decreased by 6 weeks, with no further significant change between 6 and 12 weeks post CRT (Table 2).

\section{Interval Changes Following CRT in the Lymph Nodes}

There were 18/22 lymph nodes that responded to CRT at 2 years following treatment.

The responding nodes showed a significant decrease in the mean diameter of $68 \%\left(1.4+\_0.3\right)$ and volume of $95 \%\left(8+\_1.4\right)$ at 6 weeks and a further reduction between 6 and 12 weeks $(\mathrm{p}<.0001)$.

There was no significant increase in mean values of ADCmean and ADCmedian by 6 or 12 weeks post CRT.

ADC kurtosis showed a significant mean decrease (-35\%) at 6 weeks. By 12 weeks, energy (+170\%) and CoV (+280\%) showed a significant mean increase compared to the pretreatment values whilst there was a statistically significant mean reduction in entropy $(-39 \%)$. 
TABLE 2 | Pretreatment, 6-week post-CRT and 12-week post-CRT primary tumour ADC and post-gadolinium T1w histogram parameters and their interval changes with treatment (all with treatment success $n=23$ ).

\begin{tabular}{|c|c|c|c|c|c|c|c|c|c|}
\hline $\begin{array}{l}\text { Primary tumour } \\
(n=23)\end{array}$ & Pretreatment & $\begin{array}{c}6 \text { weeks post } \\
\text { therapy }\end{array}$ & $\begin{array}{c}12 \text { weeks post } \\
\text { therapy }\end{array}$ & $\begin{array}{c}\% \text { change } \\
\text { pretreatment to } \\
6 \text { weeks }\end{array}$ & $\begin{array}{c}\mathrm{p}- \\
\text { value }\end{array}$ & $\begin{array}{c}\% \text { change } \\
\text { pretreatment to } \\
12 \text { weeks }\end{array}$ & $\begin{array}{c}\mathrm{p}- \\
\text { value }\end{array}$ & $\begin{array}{c}\% \text { change } 6 \text { to } \\
12 \text { weeks }\end{array}$ & $\begin{array}{c}\mathrm{p}- \\
\text { value }\end{array}$ \\
\hline Parameter & Median (IQR) & Median (IQR) & Median (IQR) & & & & & & \\
\hline Diameter (cm) & $\begin{array}{c}2.36 \\
(1.756-2.71)\end{array}$ & $\begin{array}{c}0.51 \\
(1.12-0.61)\end{array}$ & $\begin{array}{c}0.66 \\
(0.59-1.74)\end{array}$ & -71 & $<0.001$ & -71 & $<0.001$ & -2 & 0.07 \\
\hline Volume $\left(\mathrm{cm}^{3}\right)$ & $\begin{array}{c}6.91 \\
(316.64- \\
533.74)\end{array}$ & $\begin{array}{c}0.07 \\
(0.75-0.11)\end{array}$ & $\begin{array}{c}0.15 \\
(0.11-2.76)\end{array}$ & -97 & $<0.001$ & -98 & $<0.001$ & -6 & 0.07 \\
\hline \multicolumn{10}{|l|}{$A D C\left(\times 10^{-6} \mathrm{~mm}^{2} / \mathrm{s}\right)$} \\
\hline Mean & 729.10 & 1019.68 & $\begin{array}{c}1165.74 \\
(1006.66- \\
1197.93)\end{array}$ & +40 & $<0.001$ & +60 & $<0.001$ & +14 & 0.12 \\
\hline Median & 692.75 & 1062.5 & 1169.25 & +53 & $<0.001$ & +69 & $<0.001$ & +10 & 0.11 \\
\hline Skewness & 0.30 & -0.024 & 0.06 & -108 & 0.02 & -78 & 0.05 & -370 & 0.18 \\
\hline Kurtosis & 3.52 & 2.05 & 1.99 & -42 & $<0.001$ & -43 & $<0.001$ & -3 & 0.21 \\
\hline CoV & 0.23 & 0.12 & 0.10 & -46 & $<0.001$ & -54 & $<0.001$ & -16 & 0.63 \\
\hline Entropy & 4.26 & 2.39 & 2.50 & -44 & $<0.001$ & -41 & $<0.001$ & +5 & 0.30 \\
\hline Energy & 0.06 & 0.21 & 0.18 & +238 & $<0.001$ & +192 & $<0.001$ & -14 & 0.19 \\
\hline \multicolumn{10}{|l|}{$\begin{array}{l}\text { T1w post-gadolinium } \\
\text { signal intensity }\end{array}$} \\
\hline Mean & $\begin{array}{c}430.27 \\
(316.648- \\
533.74)\end{array}$ & $\begin{array}{c}416.06 \\
(320.99- \\
496.55)\end{array}$ & $\begin{array}{c}437.457 \\
(365.065- \\
503.834)\end{array}$ & -3 & 0.25 & +2 & 0.73 & +5 & 0.2 \\
\hline Median & $\begin{array}{c}439.5 \\
(303.25- \\
532.50)\end{array}$ & $\begin{array}{c}408.25 \\
(321.00- \\
489.25)\end{array}$ & $\begin{array}{c}433.5 \\
(370.62-503.87)\end{array}$ & -7 & 0.31 & -1 & 0.77 & +6 & 0.14 \\
\hline Skewness & $\begin{array}{c}0.05 \\
(-0.43-0.51)\end{array}$ & $\begin{array}{c}0.09 \\
(-0.39-0.32)\end{array}$ & $\begin{array}{c}0.11 \\
(-0.09-0.39)\end{array}$ & +66 & 0.78 & +93 & 0.95 & +17 & 0.76 \\
\hline Kurtosis & $\begin{array}{c}3.24 \\
(2.67-3.55)\end{array}$ & $\begin{array}{c}2.78 \\
(2.43-3.02)\end{array}$ & $\begin{array}{c}2.75 \\
(2.46-3.29)\end{array}$ & -14 & 0.19 & -15 & 0.10 & -1 & 0.73 \\
\hline $\mathrm{CoV}$ & $\begin{array}{c}0.19 \\
(0.11-0.22)\end{array}$ & $\begin{array}{c}0.11 \\
(0.10-0.18)\end{array}$ & $\begin{array}{c}0.12 \\
(0.09-0.16)\end{array}$ & -41 & 0.05 & -36 & 0.05 & +8 & 0.64 \\
\hline Entropy & $\begin{array}{c}4.14 \\
(3.93-4.30)\end{array}$ & $\begin{array}{c}4.41 \\
(3.99-4.50)\end{array}$ & $\begin{array}{c}4.37 \\
(4.23-4.52)\end{array}$ & +6 & 0.11 & +5 & 0.01 & -1 & 0.28 \\
\hline Energy & $\begin{array}{c}0.068 \\
(0.06-0.08)\end{array}$ & $\begin{array}{c}0.05 \\
(0.05-0.07)\end{array}$ & $\begin{array}{c}0.05 \\
(0.05-0.06)\end{array}$ & -16 & 0.07 & -16 & 0.01 & +0 & 0.18 \\
\hline GLCM contrast & $\begin{array}{c}144.30 \\
(91.13-177.02)\end{array}$ & $\begin{array}{c}297.42 \\
(230.82- \\
441.22)\end{array}$ & $\begin{array}{c}291.61 \\
(250.77-338.30)\end{array}$ & +106 & $<0.001$ & +102 & $<0.001$ & -2 & 0.31 \\
\hline GLCM autocorrelation & $\begin{array}{c}1141.86 \\
(1093.48- \\
1170.03)\end{array}$ & $\begin{array}{c}1051.333 \\
(879.078- \\
1403.38)\end{array}$ & $\begin{array}{c}971.88 \\
(873.51- \\
1091.60)\end{array}$ & -8 & 0.82 & -15 & 0.02 & -8 & 0.46 \\
\hline GLCM entropy & $\begin{array}{c}6.79 \\
(6.46-7.08)\end{array}$ & $\begin{array}{c}4.35 \\
(3.66-4.71)\end{array}$ & $\begin{array}{c}4.35 \\
(4.19-4.49)\end{array}$ & -36 & $<0.001$ & -36 & $<0.001$ & +0 & 0.28 \\
\hline GLCM cluster shade & $\begin{array}{c}203.63 \\
(-2503.10- \\
3129.10)\end{array}$ & $\begin{array}{c}1010.87 \\
(-4628.71- \\
5465.97)\end{array}$ & $\begin{array}{c}1402.37 \\
(11.05-4029.17)\end{array}$ & +396 & 0.99 & +589 & 0.93 & +39 & 0.68 \\
\hline
\end{tabular}

$p<0.001$ considered statistically significant.

None of the post gad T1w histogram parameters showed significant change with treatment. However, mean GLCM contrast significantly increased and mean GLCM entropy significantly decreased $(+61 \%$ and $-20 \%)$ by 6 weeks post CRT with no further significant alteration between 6 and 12 weeks (Table 3).

The non-responding metastatic lymph nodes did not demonstrate a significant change in any ADC or post gadolinium T1w histogram or GLCM parameters between pretreatment and post-CRT studies. They also did not show a significant decrease in diameter or volume (Table 4).

\section{DISCUSSION}

\section{Summary of Findings}

Interval changes in signal heterogeneity have been evaluated to provide insight into the effect of CRT on the primary tumour and lymph nodes in HNSCC, and to identify whether heterogeneity changes might provide an earlier indication of treatment response. Changes in selected histogram parameters were observed by 6 weeks on ADC maps in responding primary tumours (kurtosis, CoV, entropy, energy) and responding 
TABLE 3 | Pretreatment, 6-week post-CRT and 12-week post-CRT ADC and post-gadolinium T1w texture parameters and their interval changes in metastatic lymph nodes of participants with treatment response $(n=18)$.

\begin{tabular}{|c|c|c|c|c|c|c|c|c|c|}
\hline $\begin{array}{l}\text { Largest node } \\
(n=18)\end{array}$ & Pretreatment & $\begin{array}{c}6 \text { weeks post } \\
\text { therapy }\end{array}$ & $\begin{array}{c}12 \text { weeks post } \\
\text { therapy }\end{array}$ & $\begin{array}{c}\% \text { change } \\
\text { pretreatment to } \\
6 \text { weeks }\end{array}$ & $\begin{array}{c}\mathrm{p}- \\
\text { value }\end{array}$ & $\begin{array}{c}\% \text { change } \\
\text { pretreatment to } \\
12 \text { weeks }\end{array}$ & $\begin{array}{c}\mathrm{p}- \\
\text { value }\end{array}$ & $\begin{array}{c}\% \text { change } 6 \text { to } \\
12 \text { weeks }\end{array}$ & $\begin{array}{c}\mathrm{p}- \\
\text { value }\end{array}$ \\
\hline Parameter & Median (IQR) & Median (IQR) & Median (IQR) & & & & & & \\
\hline Diameter (cm) & $\begin{array}{c}1.92 \\
(1.45-2.79)\end{array}$ & $\begin{array}{c}0.60 \\
(0.59-1.03)\end{array}$ & $\begin{array}{c}0.58 \\
(0.59-0.68)\end{array}$ & -69 & $<0.001$ & -70 & $<0.001$ & -4 & 1 \\
\hline Volume $\left(\mathrm{cm}^{3}\right)$ & $\begin{array}{c}3.74 \\
(1.61-11.46)\end{array}$ & $\begin{array}{c}0.19 \\
(0.11-0.58)\end{array}$ & $\begin{array}{c}0.11 \\
(0.10-0.17)\end{array}$ & -95 & $<0.001$ & -96 & $<0.001$ & -39 & 1 \\
\hline \multicolumn{10}{|l|}{$\operatorname{ADC}\left(\mathrm{mm}^{2} / \mathrm{s}\right)$} \\
\hline Mean & $\begin{array}{c}745.42 \\
(634.19- \\
957.6)\end{array}$ & $\begin{array}{c}824.68 \\
(686.46- \\
971.37)\end{array}$ & $\begin{array}{c}980.27 \\
(868.44- \\
1130.14)\end{array}$ & +11 & 0.30 & +32 & 0.01 & +19 & 0.12 \\
\hline Median & $\begin{array}{c}821.08 \\
(0.19-0.34)\end{array}$ & $\begin{array}{c}856.5 \\
(1.86-2.58)\end{array}$ & $\begin{array}{c}972.5 \\
(821.12- \\
1126.50)\end{array}$ & +4 & 0.17 & +18 & 0.01 & +14 & 0.11 \\
\hline Skewness & $\begin{array}{c}0.30 \\
(-0.10-0.72)\end{array}$ & $\begin{array}{c}0.06 \\
(-0.19-0.31)\end{array}$ & $\begin{array}{c}-0.02 \\
(-0.28-0.21)\end{array}$ & -78 & 0.01 & -108 & 0.10 & -138 & 0.18 \\
\hline Kurtosis & $\begin{array}{c}3.60 \\
(2.96-5.22)\end{array}$ & $\begin{array}{c}2.35 \\
(1.93-2.87)\end{array}$ & $\begin{array}{c}2.19 \\
(1.86-2.58)\end{array}$ & -35 & $<0.001$ & -39 & $<0.001$ & -7 & 0.21 \\
\hline $\mathrm{CoV}$ & $\begin{array}{c}0.23 \\
(0.19-0.29)\end{array}$ & $\begin{array}{c}0.12 \\
(0.09-0.19)\end{array}$ & $\begin{array}{c}0.10 \\
(0.09-0.13)\end{array}$ & +60 & 0.01 & +280 & $<0.001$ & -16 & 0.63 \\
\hline Entropy & $\begin{array}{c}4.09 \\
(3.74-4.37)\end{array}$ & $\begin{array}{c}3.26 \\
(2.50-4.12)\end{array}$ & $\begin{array}{c}2.50 \\
(2.17-3.12)\end{array}$ & -20 & 0.01 & -39 & $<0.001$ & -23 & 0.30 \\
\hline Energy & $\begin{array}{c}0.06 \\
(0.05-0.10)\end{array}$ & $\begin{array}{c}0.18 \\
(0.16-0.22)\end{array}$ & $\begin{array}{c}0.21 \\
(0.18-0.24)\end{array}$ & +170 & 0.01 & +170 & $<0.001$ & +14 & 0.187 \\
\hline \multicolumn{10}{|l|}{$\begin{array}{l}\text { T1w post-gadolinium } \\
\text { signal intensity }\end{array}$} \\
\hline Mean & $\begin{array}{c}435.8 \\
(363.51- \\
510.12)\end{array}$ & $\begin{array}{c}441.74 \\
(341.51- \\
578.21)\end{array}$ & $\begin{array}{c}502.53 \\
(308.77-537.53)\end{array}$ & +1 & 0.72 & +15 & 0.07 & +14 & 0.19 \\
\hline Median & $\begin{array}{c}435.9 \\
(347-52)\end{array}$ & $\begin{array}{c}443.68 \\
(334.50- \\
584.00)\end{array}$ & $\begin{array}{c}506.39 \\
(307.25-537.75)\end{array}$ & +2 & 0.81 & +16 & 0.07 & +14 & 0.19 \\
\hline Skewness & $\begin{array}{c}-0.14 \\
(-0.74-0.38)\end{array}$ & $\begin{array}{c}-0.007 \\
(-0.56-0.51)\end{array}$ & $\begin{array}{c}0.08 \\
(-0.16-0.23)\end{array}$ & -95 & 0.42 & +39 & 0.81 & +1342 & 0.88 \\
\hline Kurtosis & $\begin{array}{c}3.4 \\
(2.37-4.12)\end{array}$ & $\begin{array}{c}3.30 \\
(2.56-3.5)\end{array}$ & $\begin{array}{c}3.23 \\
(2.588-3.7)\end{array}$ & -3 & 0.72 & -4 & 0.58 & -2 & 0.83 \\
\hline CoV & $\begin{array}{c}0.71 \\
(0.67-0.70)\end{array}$ & $\begin{array}{c}0.78 \\
(0.69-0.84)\end{array}$ & $\begin{array}{c}0.93 \\
(0.74-0.94)\end{array}$ & +10 & 0.00 & +31 & 0 & +19 & 0.47 \\
\hline Entropy & $\begin{array}{c}4.2 \\
(4.03-4.39)\end{array}$ & $\begin{array}{c}4.41 \\
(4.205-4.62)\end{array}$ & $\begin{array}{c}4.31 \\
(3.88-4.45)\end{array}$ & +5 & 0.03 & +3 & 0.07 & -2 & 0.19 \\
\hline Energy & $\begin{array}{c}0.06 \\
(0.05-0.07)\end{array}$ & $\begin{array}{c}0.05 \\
(0.04-0.06)\end{array}$ & $\begin{array}{c}0.06 \\
(0.05-0.08)\end{array}$ & -44 & 0.04 & -14 & 0.05 & +7 & 0.20 \\
\hline GLCM contrast & 145.5 & $\begin{array}{c}234.89 \\
(157.75- \\
319.80)\end{array}$ & 264.33 & +61 & $<0.001$ & +82 & $<0.001$ & +13 & 0.46 \\
\hline GLCM autocorrelation & $\begin{array}{c}1114.34 \\
(1054.0- \\
1161.4)\end{array}$ & $\begin{array}{l}1146.76 \\
(893.15- \\
1398.21)\end{array}$ & $\begin{array}{c}1168.3 \\
(893.15- \\
1398.21)\end{array}$ & -3 & 0.34 & -5 & 0.06 & +2 & 0.73 \\
\hline GLCM entropy & $\begin{array}{c}6.96 \\
(6.60-7.18)\end{array}$ & $\begin{array}{c}5.55 \\
(4.48-5.96)\end{array}$ & $\begin{array}{c}4.42 \\
(4.19-4.57)\end{array}$ & -20 & $\begin{array}{c}< \\
0.001\end{array}$ & -36 & $<0.001$ & -20 & 0.002 \\
\hline GLCM cluster shade & $\begin{array}{c}404.66 \\
(-4224.62- \\
2081.77)\end{array}$ & $\begin{array}{c}385.24 \\
(-2066.94- \\
2792.61)\end{array}$ & $\begin{array}{c}584.18 \\
(-2234.55- \\
2116.94)\end{array}$ & -195 & 0.44 & +244 & 0.62 & +52 & 0.58 \\
\hline
\end{tabular}

$p<0.001$ considered statistically significant.

lymph nodes (kurtosis). Whilst there were no post CRT changes in histogram parameters on post gad T1w images, there were significant decreases in GLCM entropy for both successfully treated primary tumours and lymph nodes. The nonresponding lymph nodes did not demonstrate any of these interval changes in heterogeneity. Interval reduction in size was also demonstrated by 6 weeks in responding lymph nodes but not in non-responding lymph nodes.

\section{ADC}

Previous studies have evaluated the role of diffusion-weighted MRI in predicting HNSCC treatment response post CRT, and 
TABLE 4 | Pretreatment, 6-week post-CRT and 12-week post-CRT ADC and post-gadolinium T1w histogram parameters and their interval changes in metastatic lymph nodes of participants with treatment failure $(n=4)$.

\begin{tabular}{|c|c|c|c|c|c|c|c|c|c|}
\hline $\begin{array}{l}\text { Node non-responders } \\
(n=4)\end{array}$ & Pretreatment & $\begin{array}{c}6 \text { weeks post } \\
\text { therapy }\end{array}$ & $\begin{array}{c}12 \text { weeks post } \\
\text { therapy }\end{array}$ & $\begin{array}{c}\% \text { change } \\
\text { pretreatment to } \\
6 \text { weeks }\end{array}$ & $\begin{array}{c}\mathrm{p}- \\
\text { value }\end{array}$ & $\begin{array}{c}\% \text { change } \\
\text { pretreatment to } \\
12 \text { weeks }\end{array}$ & $\begin{array}{c}\mathrm{p}- \\
\text { value }\end{array}$ & $\begin{array}{c}\% \text { change } 6 \text { to } 12 \\
\text { weeks }\end{array}$ & $\begin{array}{c}\text { p- } \\
\text { value }\end{array}$ \\
\hline Parameter & Median (IQR) & Median (IQR) & Median (IQR) & & & & & & \\
\hline Effective diameter (cm) & $\begin{array}{c}2.2 \\
(1.23-3.5)\end{array}$ & $\begin{array}{c}1.1 \\
(0.69-1.55)\end{array}$ & $\begin{array}{c}0.6 \\
(0.50-1.35)\end{array}$ & -50 & 0.14 & -71 & 0.14 & -45 & 0.14 \\
\hline Volume $\left(\mathrm{cm}^{3}\right)$ & $\begin{array}{c}8.6 \\
(1.35-19.59)\end{array}$ & $\begin{array}{c}0.9 \\
(0.19-2.11)\end{array}$ & $\begin{array}{c}0.1 \\
(0.07-1.65)\end{array}$ & -90 & 0.14 & -98 & 0.14 & -89 & 0.12 \\
\hline \multicolumn{10}{|l|}{$\operatorname{ADC}\left(\mathrm{mm}^{2} / \mathrm{s}\right)$} \\
\hline Mean & $\begin{array}{c}687 \\
(580.90- \\
876.15)\end{array}$ & $\begin{array}{c}932 \\
(647.52- \\
1119.22)\end{array}$ & $\begin{array}{c}949.2 \\
(848.36- \\
1208.84)\end{array}$ & 36 & 0.14 & 57 & 0.14 & 2 & 0.62 \\
\hline Median & $\begin{array}{c}698.3 \\
(576.00- \\
884.25)\end{array}$ & $\begin{array}{c}918.3 \\
(640.25- \\
1134.50)\end{array}$ & $\begin{array}{c}969.3 \\
(835.38- \\
1203.63)\end{array}$ & 32 & 0.14 & 53 & 0.14 & 6 & 0.87 \\
\hline Skewness & $\begin{array}{c}0.6 \\
(-0.22-2.23)\end{array}$ & $\begin{array}{c}-0.1 \\
(-0.69-0.42)\end{array}$ & $\begin{array}{c}0.3 \\
(-0.46-0.66)\end{array}$ & -117 & 0.46 & -400 & 0.46 & -400 & 0.62 \\
\hline Kurtosis & $\begin{array}{c}5.9(2.21- \\
12.76)\end{array}$ & $2.8(2.00-3.70)$ & $2.3(2.08-2.81)$ & -53 & 0.71 & -18 & 0.27 & -18 & 0.62 \\
\hline $\mathrm{CoV}$ & $\begin{array}{l}0.3(0.17- \\
0.55)\end{array}$ & $0.2(0.17-0.26)$ & $\begin{array}{c}0.218(0.14- \\
0.24)\end{array}$ & -33 & 0.46 & -8 & 0.27 & 9 & 0.62 \\
\hline Entropy & $\begin{array}{c}4.1(3.52- \\
4.49)\end{array}$ & 3.7 (2.78-4.29) & $2.0(1.52-4.08)$ & -10 & 0.14 & -52 & 0.14 & -46 & 0.25 \\
\hline Energy & $\begin{array}{c}0.06 \\
(0.05-0.12)\end{array}$ & $\begin{array}{c}0.08 \\
(0.06-0.18)\end{array}$ & $\begin{array}{c}0.3 \\
(0.08-0.37)\end{array}$ & 60 & 0.14 & 200 & 0.14 & 275 & 0.25 \\
\hline \multicolumn{10}{|l|}{$\begin{array}{l}\text { T1w post-gadolinium } \\
\text { signal intensity }\end{array}$} \\
\hline Mean & $\begin{array}{c}473.98 \\
(276.67- \\
548.19)\end{array}$ & $\begin{array}{c}436.02 \\
(244.55- \\
595.17)\end{array}$ & $\begin{array}{c}442.05 \\
(308.77-537.53)\end{array}$ & -2 & 0.71 & -1 & 1 & 1 & 0.87 \\
\hline Median & $\begin{array}{c}499.5 \\
(277.25- \\
557.50)\end{array}$ & $\begin{array}{c}440 \\
(243.25- \\
598.50)\end{array}$ & $\begin{array}{c}439 \\
(307.25-537.75)\end{array}$ & -4 & 0.71 & -4 & 1 & 0 & 0.87 \\
\hline Skewness & $\begin{array}{c}0.09 \\
(-0.50-0.23)\end{array}$ & $\begin{array}{c}-0.1 \\
(-0.27-0.22)\end{array}$ & $\begin{array}{c}0.08 \\
(-0.16-0.23)\end{array}$ & -12 & 0.71 & -186 & 0.71 & -198 & 0.61 \\
\hline Kurtosis & $\begin{array}{c}2.83 \\
(2.44-3.85)\end{array}$ & $\begin{array}{c}2.82 \\
(2.74-3.46)\end{array}$ & $\begin{array}{c}3.34 \\
(2.58-3.72)\end{array}$ & -1 & 0.71 & 6 & 0.46 & 7 & 0.14 \\
\hline CoV & $\begin{array}{c}0.81 \\
(0.67-47.90)\end{array}$ & $\begin{array}{c}0.78 \\
(0.69-0.84)\end{array}$ & $\begin{array}{c}0.83 \\
(0.744-0.94)\end{array}$ & -4 & 0.71 & 2 & 0.46 & 6 & 0.14 \\
\hline Entropy & $\begin{array}{c}4.26 \\
(3.99-4.47)\end{array}$ & $\begin{array}{c}4.43 \\
(4.23-4.56)\end{array}$ & $\begin{array}{c}4.28 \\
(3.88-4.45)\end{array}$ & 4 & 0.47 & -1 & 0.71 & -5 & 0.87 \\
\hline Energy & $\begin{array}{c}0.06 \\
(0.05-0.07)\end{array}$ & $\begin{array}{c}0.05 \\
(0.04-0.06)\end{array}$ & $\begin{array}{c}0.06 \\
(0.05-0.080\end{array}$ & -13 & 0.47 & 3 & 1 & 18 & 0.72 \\
\hline GLCM contrast & $\begin{array}{c}0.97 \\
(0.84-1.18)\end{array}$ & $\begin{array}{c}4.58 \\
(4.34-5.22)\end{array}$ & $\begin{array}{c}300.09 \\
(168.86-412.19)\end{array}$ & +372 & 0.07 & +30837 & 0.07 & +6452 & 0.07 \\
\hline GLCM autocorrelation & $\begin{array}{c}6.64 \\
(5.96-6.98)\end{array}$ & $\begin{array}{c}973.99 \\
(879.09- \\
1152.96)\end{array}$ & $\begin{array}{c}4.37 \\
(4.18-6.05)\end{array}$ & $+14,555$ & 0.07 & -34 & 0.14 & -100 & 0.07 \\
\hline GLCM entropy & $\begin{array}{c}473.98 \\
(276.67- \\
548.19)\end{array}$ & $\begin{array}{c}436.02 \\
(244.55- \\
595.17)\end{array}$ & $\begin{array}{c}442.05 \\
(308.77-537.53)\end{array}$ & +841 & 0.07 & -99 & 0.07 & -100 & 0.07 \\
\hline GLCM cluster shade & $\begin{array}{c}499.5 \\
(277.25- \\
557.50)\end{array}$ & $\begin{array}{c}440 \\
(243.25- \\
598.50)\end{array}$ & $\begin{array}{c}439 \\
(307.25-537.75)\end{array}$ & -126 & 0.07 & -9 & 1 & -456 & 0.07 \\
\hline
\end{tabular}

most have found that a greater rise in ADCmean from the pretreatment to the intra-treatment (15-19) or posttreatment MRI $(20,21)$ predicts treatment outcomes. Similarly, our study demonstrated that primary tumour ADCmean increased by 6 weeks post CRT. However, interval changes in ADCmean did not differ between metastatic lymph nodes that did and did not respond to treatment. This may be due to our inclusion of necrotic areas in the ROIs, which could have led to an overestimation of baseline ADCmean.

King et al. found that ADC histogram analysis could predict intra-treatment response at the primary site as early as 2 weeks after the start of treatment, with primary tumours showing higher histogram skewness (20). de Parrot et al. demonstrated that the ADC histogram kurtosis ratio (22) was useful in 
differentiating histologic grades of HNSCC at $b=2,000 \mathrm{~s} / \mathrm{mm}^{2}$. A general shift in skewness to the left means that the tail of the ADC curves got heavier toward the negative and signifies a greater asymmetry of the data posttreatment. Similar findings were reported by Forouton et al. (23), who demonstrated negative skewness and greater asymmetry following therapy in the treatment group when compared to the control group. Whilst our data showed a non-significant increase in skewness post CRT, the histogram parameters of kurtosis, coefficient of variation and energy were found to demonstrate a significant increase. This was demonstrated by 6-week post CRT for primary tumour and 12 weeks post CRT for lymph nodes. The underlying histopathological basis for this is yet to be studied; however, it appears there is a trend toward greater homogeneity of the tumour with treatment.

\section{Post-Gadolinium T1w MRI}

There are limited data on the interval changes in macroscopic T1w gadolinium enhancement following CRT for HNSCC. A qualitative grading (24) showed that changes in the pattern of necrosis on T1w gadolinium enhancement imaging could predict the outcome. The GLCM is a tabulation of how often different combinations of grey levels occur between neighbouring pixels in an image (21-23). Reinert et al. (25) found that GLCM entropy significantly decreased, whereas GLCM uniformity significantly increased $(\mathrm{p}<.001)$ after therapy in a study assessing response of Hodgkin lymphoma to chemotherapy. Whilst none of the histogram parameters changed significantly post CRT in our cohort, GLCM contrast increased whilst GLCM entropy decreased with treatment in both the primary tumour and lymph node by 6 weeks post CRT. It is of interest that changes were observed in similar parameters in both metastatic lymph nodes (largely non-enhancing necrosis in $16 / 18$ pretreatment) and primary tumours (homogenously enhancing in $22 / 23$ pretreatment) considering the marked difference in pretreatment enhancement patterns.

\section{Differences Between the Primary Tumour and Nodes}

The interval changes in ADC-based histogram parameters differed between the primary tumours and metastatic lymph nodes, with earlier changes being observed with primary tumours. Although there are no previous comparable data available for ADC-based histogram parameters, a potential disparity between the timing and nature of posttreatment ADC-related biomarkers between the primary tumour and nodes has been established in previous studies. In particular, these have demonstrated that the ADCmean acquired from nodal sites is able to predict treatment outcomes, whereas ADCs acquired from primary sites do not (26), whilst predictive threshold ADC measurements differ between primary tumour and nodal sites (27).

\section{Comparison With Primary Tumour and Nodal Size and Volume Change}

The assessment of morphological features such as changes in tumour dimensions has provided prognostic stratification (20,
$24,28,29)$ in the posttreatment setting, but they have variable diagnostic accuracy in determining treatment response $(30,31)$. A significant reduction in lymph node dimensions at 6 weeks post CRT was only shown in those with successful treatment. However, it is of interest that the difference in magnitude between responding and non-responding lymph node dimensions was less than that demonstrated with some heterogeneity parameters. The decrease in diameter and volume of the responding lymph nodes at 6 weeks post CRT was $69 \%$ and $95 \%$ as compared to $50 \%$ and $90 \%$ with the nonresponding lymph nodes. Thus, the addition of histogram parameters could perhaps aid in increasing the discriminatory power of size criteria in assessing posttreatment changes, even as early as 6 weeks posttreatment.

\section{Limitations}

It is acknowledged that there are some limitations to this study.

Firstly, it should be noted that the cohort was largely represented by HPV-positive oropharyngeal cancer. HPV-OPC is a clinically and histologically distinct form of HNSCC which has a better outcome irrespective of treatment choice. Since it exhibits particular histopathological features such as indistinct cell borders and comedo-necrosis, there should be caution in extrapolation of the results to a wider HNSCC population.

Secondly, there was only a small sample of non-responders to CRT with only four metastatic lymph nodes and no primary tumours which did not respond to treatment. The small sample of non-responding lymph nodes precluded a direct statistical comparison of parameters between responding and nonresponding lymph nodes. It also raises the possibility that interval changes in histogram and GLCM parameters in the non-responding lymph nodes were not detected due to a type 2 error. Future studies should be conducted with a larger HPV negative cohort and a greater proportion of non-responders such that these texture parameters can be evaluated for their ability to predict treatment response.

Finally, it could be argued that the outcomes were influenced by our method of ROI analysis which was focused on whole volumes of the primary tumour or lymph node. Both volume and area-based ROI data are variably presented in the literature. However, there are limited data on how this impacts on histogram or GLCM analysis. Generous sampling of the whole volume may better reflect tumour heterogeneity. However, smaller ROIs benefit from the ability to exclude macroscopic necrosis. It is possible that sampling certain parameters such as ADCmean may have revealed significant changes if confined to enhancing "viable tumour", particularly with respect to the largely necrotic lymph nodes.

\section{CONCLUSIONS}

This exploratory study indicates that significant post-CRT interval changes in histogram and GLCM parameters are demonstrated on ADC maps and T1w post gad images as early as 6 weeks following successful treatment for stage III and 
IV HNSCC. The selection of appropriate ADC map histogram parameters may differ, depending on whether biomarkers for primary tumour or lymph node response are being evaluated. A small sample of non-responding metastatic lymph nodes did not demonstrate such interval evolution of these heterogeneity parameters; however, standard size criteria were also able to predict treatment failure in these patients.

\section{DATA AVAILABILITY STATEMENT}

The data that support the findings of this study are available from the corresponding author upon reasonable request.

\section{ETHICS STATEMENT}

Institutional approval from the Research Ethics Committee (REC reference 13/LO/1876) and informed consent was obtained from all participants. The study conforms to recognized standards of the Declaration of Helsinki.

\section{AUTHOR CONTRIBUTIONS}

Conceptualization, SC, VG; Methodology, SC, VG, GC; Investigation, SC, AG, MA; Data Curation, AG, SC; Writing Original Draft, AG, SC; Writing - Review \& Editing, VG, GC;

\section{REFERENCES}

1. Ferlay J, Soerjomataram I, Dikshit R, Esers S, Mathers C, Rebelo M, et al. Cancer Incidence and Mortality Worldwide: Sources, Methods and Major Patterns in GLOBOCAN 2012. Int J Cancer (2015) 136(5):E359-86. doi: $10.1002 /$ ijc. 29210

2. Bernier J, Pfister DG, Cooper JS. Adjuvant Chemo- and Radiotherapy for Poor Prognosis Head and Neck Squamous Cell Carcinomas. Crit Rev Oncol Hematol (2005) 56(3):353-64. doi: 10.1016/j.critrevonc.2005.04.010

3. Hauswald H, Simon C, Hecht S, Debus J, Lindel K. Long-Term Outcome and Patterns of Failure in Patients With Advanced Head and Neck Cancer. Radiat Oncol (2011) 6:70. doi: 10.1186/1748-717X-6-70

4. Mabanta SR, Mendenhall WM, Stringer SP, Cassisi NJ. Salvage Treatment for Neck Recurrence After Irradiation Alone for Head and Neck Squamous Cell Carcinoma With Clinically Positive Neck Nodes. Head Neck (1999) 21 (7):591-4. doi: 10.1002/(SICI)1097-0347(199910)21:7<591::AIDHED1>3.0.CO;2-Y

5. Varoquaux A, Rager O, Dulguerov P, Burkhardt K, Ailianou A, Becker M. Diffusion-Weighted and PET/MR Imaging After Radiation Therapy for Malignant Head and Neck Tumors. RadioGraphics (2015) 35(5):1502-27. doi: 10.1148/rg.2015140029

6. Subramaniam RM, Truong M, Peller P, Sakai O, Mercier G. Fluorodeoxyglucose-Positron-Emission Tomography Imaging of Head and Neck Squamous Cell Cancer. Am J Neuroradiol (2010) 31(4):598-604. doi: 10.3174/ajnr.A1760

7. Gupta T, Master Z, Kannan S, Agarwal JP, Ghsoh-Laskar S, Rangarajan V, et al. Diagnostic Performance of Post-Treatment FDG PET or FDG PET/CT Imaging in Head and Neck Cancer: A Systematic Review and Meta-Analysis. Eur J Nucl Med Mol Imaging (2011) 38(11):2083. doi: 10.1007/s00259-011-1893-y

8. Just N. Improving Tumour Heterogeneity MRI Assessment With Histograms. Br J Cancer (2014) 111(12):2205-13. doi: 10.1038/bjc.2014.512
Supervision, SC, VG; Project Administration, SC: Funding Acquisition, SC.

\section{FUNDING}

This research was funded in whole, or in part, by the Wellcome Trust $[203148 / \mathrm{Z} / 16 / \mathrm{Z}]$. For the purpose of open access, the author has applied a CC BY public copyright licence to any Author Accepted Manuscript version arising from this submission. Authors acknowledge funding support from Wellcome/Engineering and Physical Sciences Research Council Centre for Medical Engineering at King's College London (WT 203148/Z/16/Z); National Institute for Health Research Biomedical Research Centre at Guy's \& St Thomas' Hospitals and King's College London; Cancer Research UK National Cancer Imaging Translational Accelerator (A27066); the UK Research \& Innovation London Medical Imaging and Artificial Intelligence Centre. Authors also acknowledge funding suport from Guy's and St Thomas' Hospital Charity (ref EFT130501) and the Royal College of Radiologists: Kodak Radiology Fund Research Bursary.

\section{SUPPLEMENTARY MATERIAL}

The Supplementary Material for this article can be found online at: https://www.frontiersin.org/articles/10.3389/fonc.2022.784693/ full\#supplementary-material

9. Kim YJ, Kim SH, Lee AW, Jin M-S, Kang BJ, Song BJ. Histogram Analysis of Apparent Diffusion Coefficients After Neoadjuvant Chemotherapy in Breast Cancer. Jpn J Radiol (2016) 34(10):657-66. doi: 10.1007/s11604-016-0570-2

10. King AD, Chow K-K, Yu K-H, Mo FKF, Yeung DKW, Yuan J, et al. Head and Neck Squamous Cell Carcinoma: Diagnostic Performance of DiffusionWeighted MR Imaging for the Prediction of Treatment Response. Radiology (2013) 266(2):531-8. doi: 10.1148/radiol.12120167

11. Ren J-L, Yuan Y, Li X-X, Shi Y-Q, Tao X-F. Histogram Analysis of Apparent Diffusion Coefficient Maps in the Prognosis of Patients With Locally Advanced Head and Neck Squamous Cell Carcinoma: Comparison of Different Region of Interest Selection Methods. Eur J Radiol (2018) 106:713. doi: 10.1016/j.ejrad.2018.07.004

12. Jacob R, Welkoborsky HJ, Mann WJ, Höfken F, Dienes HP, Freije JE. Heterogeneity of Squamous Cell Carcinomas of the Head and NeckAnalysis of Tumor Biologic Factors and Proliferation Rates. Laryngoscope (1996) 106(9):1170-5. doi: 10.1097/00005537-199609000-00023

13. Veneroni S, Silvestrini R, Costa A, Salvatori P, Faranda A, Molinari R. Biological Indicators of Survival in Patients Treated by Surgery for Squamous Cell Carcinoma of the Oral Cavity and Oropharynx. Oral Oncol (1997) 33(6):408-13. doi: 10.1016/S0964-1955(97)00036-5

14. Zwanenburg A, Vallières M, Abdalah MA, Aerts HJWL, Andrearczyk V, Apte $A$, et al. The Image Biomarker Standardization Initiative: Standardized Quantitative Radiomics for High-Throughput Image-Based Phenotyping. Radiology (2020) 295(2):328-38. doi: 10.1148/radiol.2020191145

15. Kim S, Loevner L, Quon H, Sherman E, Weinstein G, Kilger A, et al. Diffusion-Weighted Magnetic Resonance Imaging for Predicting and Detecting Early Response to Chemoradiation Therapy of Squamous Cell Carcinomas of the Head and Neck. Clin Cancer Res (2009) 15(3):986-94. doi: 10.1158/1078-0432.CCR-08-1287

16. Marzi S, Piludu F, Sanguineti G, Marucci L, Farneti A, Terrenato I, et al. The Prediction of the Treatment Response of Cervical Nodes Using Intravoxel 
Incoherent Motion Diffusion-Weighted Imaging. Eur J Radiol (2017) 92:93102. doi: 10.1016/j.ejrad.2017.05.002

17. Berrak S, Chawla S, Kim S, Quon H, Sherman E, Loevner LA, et al. Diffusion Weighted Imaging in Predicting Progression Free Survival in Patients With Squamous Cell Carcinomas of the Head and Neck Treated With Induction Chemotherapy. Acad Radiol (2011) 18(10):1225-32. doi: 10.1016/j.acra. 2011.06.009

18. Matoba M, Tuji H, Shimode Y, Toyoda I, Kuginuki Y, Miwa K, et al. Fractional Change in Apparent Diffusion Coefficient as an Imaging Biomarker for Predicting Treatment Response in Head and Neck Cancer Treated With Chemoradiotherapy. Am J Neuroradiol (2014) 35(2):379-85. doi: 10.3174/ajnr.A3706

19. Galbán CJ, Mukherji SK, Chenevert TL, Meyer CR, Hamstra DA, Bland PH, et al. A Feasibility Study of Parametric Response Map Analysis of DiffusionWeighted Magnetic Resonance Imaging Scans of Head and Neck Cancer Patients for Providing Early Detection of Therapeutic Efficacy. Transl Oncol (2009) 2(3):184-90. doi: 10.1593/tlo.09175

20. King AD, Mo FKF, Yu K-H, Yeung DKW, Zhou H, Bhatia KS, et al. Squamous Cell Carcinoma of the Head and Neck: Diffusion-Weighted MR Imaging for Prediction and Monitoring of Treatment Response. Eur Radiol (2010) 20 (9):2213-20. doi: 10.1007/s00330-010-1769-8

21. Vandecaveye V, Dirix P, De Keyzer F, Op de Beeck K, Vander Poorten V, Hauben E, et al. Diffusion-Weighted Magnetic Resonance Imaging Early After Chemoradiotherapy to Monitor Treatment Response in Head-and-Neck Squamous Cell Carcinoma. Int J Radiat Oncol (2012) 82(3):1098-107. doi: 10.1016/j.ijrobp.2011.02.044

22. de Perrot T, Lenoir V, Domingo Ayllón M, Dulguerov N, Pusztaszeri M, Becker M. Apparent Diffusion Coefficient Histograms of Human Papillomavirus-Positive and Human Papillomavirus-Negative Head and Neck Squamous Cell Carcinoma: Assessment of Tumor Heterogeneity and Comparison With Histopathology. Am J Neuroradiol (2017) 38(11):2153-60. doi: 10.3174/ajnr.A5370

23. Foroutan P, Kreahling JM, Morse DL, Grove O, Lloyd MC, Reed D, et al. Diffusion MRI and Novel Texture Analysis in Osteosarcoma Xenotransplants Predicts Response to Anti-Checkpoint Therapy. PloS One (2013) 8(12): e82875. doi: 10.1371/journal.pone.0082875

24. King AD, Yu K-H, Mo FKF, Law BKH, Yeun TWC, Bhatia KS, et al. Cervical Nodal Metastases From Head and Neck Squamous Cell Carcinoma: MRI Criteria for Treatment Assessment. Head Neck (2016) 38(S1):E1598-604. doi: 10.1002/hed.24285

25. Reinert CP, Wanek L, Bösmüller H, Federmann B, Fritz J, Sokler M, et al. Computed Tomography Texture Analysis for Assessment of Chemotherapy Response of Hodgkin Lymphoma. Med (Baltimore) (2020) 99(7):e19146-6. doi: 10.1097/MD.0000000000019146
26. Noij DP, Pouwels PJW, Ljumanovic R, Knol DL, Doornaert P, de Bree R, et al. Predictive Value of Diffusion-Weighted Imaging Without and With Including Contrast-Enhanced Magnetic Resonance Imaging in Image Analysis of Head and Neck Squamous Cell Carcinoma. Eur J Radiol (2015) 84(1):108-16. doi: 10.1016/j.ejrad.2014.10.015

27. Wong KH, Panek R, Bhide SA, Nutting CM, Harrington KJ, Newbold KL. The Emerging Potential of Magnetic Resonance Imaging in Personalizing Radiotherapy for Head and Neck Cancer: An Oncologist's Perspective. $\mathrm{Br} J$ Radiol (2017) 90(1071):20160768. doi: 10.1259/bjr.20160768

28. Bhatia KSS, King AD, Yu K-H, Vlantis AC, Tse GM, Mo FKF, et al. Does Primary Tumour Volumetry Performed Early in the Course of Definitive Concomitant Chemoradiotherapy for Head and Neck Squamous Cell Carcinoma Improve Prediction of Primary Site Outcome? Br J Radiol (2010) 83(995):964-70. doi: 10.1259/bjr/27631720

29. Labadie RF, Yarbrough WG, Weissler MC, Pillsbury HC, Mukherji SK. Nodal Volume Reduction After Concurrent Chemo- and Radiotherapy: Correlation Between Initial CT and Histopathologic Findings. Am J Neuroradiol (2000) 21 (2):310-4.

30. Clavel S, Charron M-P, Bélair M, Delouya G. The Role of Computed Tomography in the Management of the Neck After Chemoradiotherapy in Patients With Head-and-Neck Cancer. Int J Radiat Oncol Biol Phys (2012) 82 (2):567-73. doi: 10.1016/j.ijrobp.2010.11.066

31. Nishimura G, Matsuda H, Taguchi T, Takahashi M, Komatsu M, Sano D, et al. Treatment Evaluation of Metastatic Lymph Nodes After Concurrent Chemoradiotherapy in Patients With Head and Neck Squamous Cell Carcinoma. Anticancer Res (2012) 32(2):595-600.

Conflict of Interest: The authors declare that the research was conducted in the absence of any commercial or financial relationships that could be construed as a potential conflict of interest.

Publisher's Note: All claims expressed in this article are solely those of the authors and do not necessarily represent those of their affiliated organizations, or those of the publisher, the editors and the reviewers. Any product that may be evaluated in this article, or claim that may be made by its manufacturer, is not guaranteed or endorsed by the publisher.

Copyright $\odot 2022$ Guha, Anjari, Cook, Goh and Connor. This is an open-access article distributed under the terms of the Creative Commons Attribution License (CC BY). The use, distribution or reproduction in other forums is permitted, provided the original author(s) and the copyright owner(s) are credited and that the original publication in this journal is cited, in accordance with accepted academic practice. No use, distribution or reproduction is permitted which does not comply with these terms. 